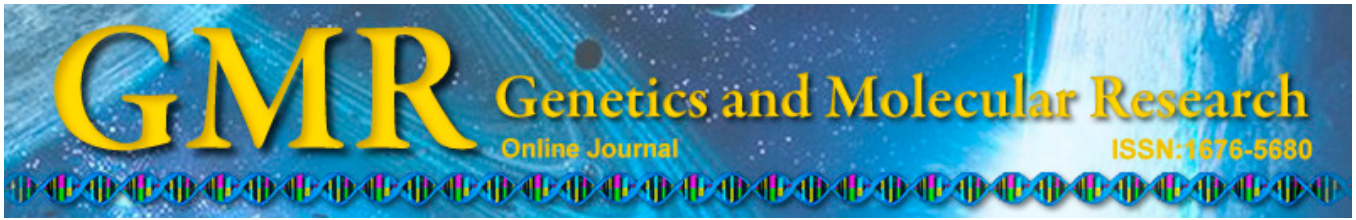

\title{
Microarray analysis of differentially expressed genes in the liver between Bai'er layers and broilers
}

\author{
Q.-G. Wang 1,2,4*, H.-F. Zhang ${ }^{1,2,3 *}$, S.-Z. Wang ${ }^{1,2,3}$, G.L. Gao ${ }^{1,2,4}$, \\ L. Leng ${ }^{1,2,3}$, W. Na ${ }^{1,2,3}$ and H. Li ${ }^{1,2,3}$ \\ ${ }^{1}$ Key Laboratory of Chicken Genetics and Breeding, Ministry of Agriculture, \\ Harbin, China \\ ${ }^{2}$ Key Laboratory of Animal Genetics, Breeding and Reproduction, \\ Education Department of Heilongjiang Province, Harbin, China \\ ${ }^{3}$ College of Animal Science and Technology, Northeast Agricultural University, \\ Harbin, China \\ ${ }^{4}$ Chongqing Academy of Animal Science, China \\ *These authors contributed equally to this study. \\ Corresponding author: $\mathrm{H}$. Li \\ E-mail: lihui@neau.edu.cn
}

Genet. Mol. Res. 14 (1): 2885-2889 (2015)

Received April 8, 2014

Accepted August 22, 2014

Published March 31, 2015

DOI http://dx.doi.org/10.4238/2015.March.31.19

\begin{abstract}
In this study, we profiled gene expression in chicken liver and screened differentially expressed genes in the Bai'er layers and fat line broilers. Birds were derived from the 14th generation of Northeast Agricultural University fat broiler lines and Bai'er layers. Chicken genome arrays were used to screen differentially expressed genes in liver tissue from the Bai'er layers and fat line broilers. We screened 671 differentially expressed genes between broilers and layers at the ages of 2 and 4 weeks. We observed enrichment of a series of significant pathways, including the mitogen-activated protein kinase signaling pathway, cell cycle, mammalian target of rapamycin signaling pathway, and p53 signaling pathway. At 2 and 4 weeks, 94 shared differentially expressed genes were observed. We
\end{abstract}


speculated that these genes regulate chicken lipid metabolism.

Key words: Chicken; Gene expression profile; Liver

\section{INTRODUCTION}

The chicken is an important model organism that bridges the evolutionary gap between mammals and other vertebrates. Excessive accumulation of lipids in adipose tissue is a major problem in the present-day broiler industry. Lipogenesis primarily occurs in the liver in avian, resulting in triglyceride (TG) accumulation in avian adipose tissue (O'Hea and Leveille, 1968). The liver may be a pivotal site for glycometabolism and lipid metabolism, as it can store glucose via glycogen synthesis and lipogenesis or release glucose via glycogenolysis and gluconeogenesis, and release lipid fuels as lipoproteins (Kotani et al., 2004). Gene chip is a high-throughput technology used to screen for differentially expressed genes screening.

In this study, chicken genome arrays were used to screen for differentially expressed genes in the liver tissue between 3 Bai'er layers and 3 fat line broilers at 2 and 4 weeks of age. Reference RNA used was an RNA pool prepared from an equal amount of total RNA from chicken liver. To better understand gene expression in the chicken liver, gene expression profiles at 2 and 4 weeks of age were investigated. This study will help elucidate the molecular mechanisms of lipid metabolism in chickens and contribute to related research in other species.

\section{MATERIAL AND METHODS}

\section{Animals}

Three fat line broilers, derived from the Northeast Agricultural University broilers lines divergently selected for abdominal fat content (NEAUHLF) (Guo et al., 2011), and 3 Bai'er layers, a Chinese local breed, were used in the current study. Birds were maintained under similar environmental conditions and had access to feed and water ad libitum. They were fed a commercial corn-soybean-based diet that met National Research Council requirements (National Research Council, 1994).

\section{Sample preparation}

Birds were slaughtered at 2 and 4 weeks of age. The liver was isolated, immediately frozen in liquid nitrogen, and stored at $-80^{\circ} \mathrm{C}$. Total RNA was isolated from livers using Trizol reagent (Invitrogen, Carlsbad, CA, USA) and quantified by spectrophotometry. mRNA was isolated using the Oligotex mRNA Mini Kit (Qiagen, Hilden, Germany). cDNA was prepared by oligo-dT-primed reverse transcription using Superscript II (Life Technologies, Carlsbad, CA, USA). cRNA probes were prepared using an IVT Labeling Kit (Affymetrix, Santa Clara, CA, USA). Microarrays were prepared using the GeneChip ${ }^{\circledR}$ Hybridization, Wash, and Stain Kit (Affymetrix). Chicken genome arrays, with comprehensive coverage of over 38,000 probe sets representing 32,773 transcripts, were created by Affymetrix.

\section{Statistical analysis}

Differentially expressed genes were identified from normalized data using the Sig- 
nificance Analysis of Microarrays algorithm (Tusher et al., 2001) and $t$-test (Forrester and Ury, 1969) in SAS (Cary, NC, USA). According to the SAM algorithm, genes were identified as differentially expressed based on expression differences among the sample groups and the consistency of these differences; a score was assigned to each gene based on its change in expression relative to the standard deviation of repeated measurements for that gene. Differences were considered highly significant when $\mathrm{P}<0.01$ and significant when $\mathrm{P}$ $<0.05$.

\section{RESULTS}

A P value of 0.05 was the screening criterion for significantly different expression. At 2 weeks of age, 882 probes representing 671 genes were differentially expressed, and at 4 weeks of age 916 probes representing 671 genes were differentially expressed.

These genes were analyzed by Gene Ontology (GO) enrichment and Kyoto Encyclopedia of Genes and Genomes (KEGG) pathways. The 671 differentially expressed genes at 2 weeks were enriched for 1 significant GO term $(\mathrm{P}<0.05)$, the ribonucleoprotein complex. Genes were mainly annotated to 15 significant pathways according to KEGG analysis $(\mathrm{P}<0.05)$, including ribosome, spliceosome, ubiquitin-mediated proteolysis, base excision repair, mitogen-activated protein kinase (MAPK) signaling pathway, aminoacyltRNA biosynthesis, gonadotropin releasing hormone signaling pathway, lysosome, glycosaminoglycan biosynthesis, protein export, p53 signaling pathway, metabolic pathways, ErbB signaling pathway, homologous recombination, mammalian target of rapamycin (mTOR) signaling pathway. A total of 671 differentially expressed genes at 4 weeks were significantly enriched for only $1 \mathrm{GO}$ term $(\mathrm{P}<0.05)$, which was the pigment metabolic process. They were annotated to 20 significant pathways according to KEGG analysis $(\mathrm{P}<$ 0.05 ), including metabolic pathways, metabolism of xenobiotics by cytochrome P450, spliceosome, steroid biosynthesis, propanoate metabolism, alanine, aspartate, and glutamate metabolism, drug metabolism - cytochrome $\mathrm{P} 450$, retinol metabolism, porphyrin and chlorophyll metabolism, peroxisome, amino sugar and nucleotide sugar metabolism, valine, leucine, and isoleucine degradation, butanoate metabolism, tyrosine metabolism, adherens junction, histidine metabolism, seleno amino acid metabolism, p53 signaling pathway, MAPK signaling pathway, and nucleotide-binding oligomerization domain-like receptor signaling pathway.

Of the differentially expressed genes, 94 genes were common in birds between the 2 ages (Table S1). Thirty-four genes were upregulated and 43 genes were downregulated in Bai'er layers compared to fat line broilers. Ten genes were upregulated at 2 weeks and downregulated at 4 weeks, while 7 genes were downregulated at 2 weeks and upregulated at 4 weeks. We used KEGG Pathway and GO Term to analyze 94 common differentially expressed genes. The 34 upregulated genes were enriched for 1 significant GO term, which was maintenance of location, and they were enriched for 4 significant pathways, including aminoacyl-tRNA biosynthesis, cell cycle, metabolic pathways, and porphyrin and chlorophyll metabolism. The 43 downregulated genes were enriched for 2 significant GO terms, including pigment metabolic process and maintenance of location, and the 43 downregulated genes were enriched for 4 significant pathway, including aminoacyl-tRNA biosynthesis, cell cycle, metabolic pathways, and porphyrin and chlorophyll metabolism. 


\section{DISCUSSION}

In the present study, genome arrays were used to identify changes in gene expression between fat line broilers and Bai'er layers at 2 and 4 weeks of age. Because de novo fatty acid synthesis in birds takes place mainly in the liver, the expression of some genes involved in lipid synthesis and secretion has been analyzed in the liver of Bai'er and fat chickens. We identified and analyzed significant pathways, such as the MAPK signaling pathway, cell cycle, mTOR signaling pathway, and p53 signaling pathway.

The MAPK signaling cascades are important for various processes in the immune response (Dong et al., 2002) and are tightly related to cell cycle control (Osaki and Gama, 2013). Recent studies have indicated that the MAPK signaling pathway in the process of adipocyte differentiation plays an important role (Wang et al., 2009).

Lipid availability is tightly associated with cell growth and cell cycle progression (Esquejo et al., 2014). Fatty acid synthesis appears to be coupled with the cell cycle via independent pathways, and inhibition of fatty acid synthesis would result in cell cycle arrest. In contrast, an early cell cycle delay was observed upon inhibition of lipid synthesis (Kwok and Wong, 2005).

In adipocytes, mTOR is thought to regulate protein synthesis (Lynch et al., 2002). Studies by Kim and Chen (2004) suggested that mTOR signaling is critical for adipocyte sensing of nutrient availability and modulating the activity of peroxisome proliferator-activated receptor- $\gamma$.

The role of p53 in regulating metabolism appears to extend well-beyond its role in tumor suppression (Maddocks and Vousden, 2011), and recent findings have linked the p53 tumor suppressor to the regulation of lipid metabolism. p53 regulates genes involved in the 3 main steps of intestinal lipid metabolism: lypolysis of dietary lipids, lipid absorption, and chylomicron packaging. p53 also regulates genes involved in hepatic lipid metabolism, the main organ orchestrating systemic lipid metabolism (Goldstein and Rotter, 2012).

In summary, our results provide a set of enriched functional pathways and genes that regulate lipid metabolism in chickens, contributing resources for the study of the molecular mechanism of fat variation between Bai'er layers and fat line broilers.

\section{ACKNOWLEDGMENTS}

The authors gratefully acknowledge the members of the Poultry Breeding Group of the College of Animal Science \& Technology, Northeast Agricultural University, for helping managing the birds and collecting the data. Research supported by National Natural Science Foundation of China (\#30972087), National “863” Project of China (\#2011AA100301), China Agriculture Research System (\#CARS-42), and Program for Innovation Research Team in University of Heilongjiang Province (\#2010td02).

\section{Supplementary material}

\section{REFERENCES}

Dong C, Davis RJ and Flavell RA (2002). MAP kinases in the immune response. Annu. Rev. Immunol. 20: 55-72.

Esquejo RM, Jeon TI and Osborne TF (2014). Lipid-cell cycle nexus: SREBP regulates microRNAs targeting Fbxw7. Cell Cycle 13: 339-340.

Forrester JC and Ury HK (1969). The Signed-Rank (Wilcoxon) test in the rapid analysis of biological data. Lancet 1: 239-241. 
Goldstein I and Rotter V (2012). Regulation of lipid metabolism by p53 - fighting two villains with one sword. Trends Endocrinol. Metab. 23: 567-575.

Guo L, Sun B, Shang Z, Leng L, et al. (2011). Comparison of adipose tissue cellularity in chicken lines divergently selected for fatness. Poultry Sci. 90: 2024-2034.

Kim JE and Chen J (2004). Regulation of peroxisome proliferator-activated receptor-gamma activity by mammalian target of rapamycin and amino acids in adipogenesis. Diabetes 53: 2748-2756.

Kotani K, Peroni OD, Minokoshi Y, Boss O, et al. (2004). GLUT4 glucose transporter deficiency increases hepatic lipid production and peripheral lipid utilization. J. Clin. Invest. 114: 1666-1675.

Kwok AC and Wong JT (2005). Lipid biosynthesis and its coordination with cell cycle progression. Plant Cell Physiol. 46: 1973-1986.

Lynch CJ, Patson BJ, Anthony J, Vaval A, et al. (2002). Leucine is a direct-acting nutrient signal that regulates protein synthesis in adipose tissue. Am. J. Physiol. Endocrinol. Metab. 283: E503-E513.

Maddocks OD and Vousden KH (2011). Metabolic regulation by p53. J. Mol. Med. 89: 237-245.

National Research Council (1994). Nutrient Requirents of Poultry. National Academy Press, Washinton.

O'Hea EK and Leveille GA (1968). Lipogenesis in isolated adipose tissue of the domestic chick (Gallus domesticus). Comp. Biochem. Physiol. 26: 111-120.

Osaki LH and Gama P (2013). MAPK signaling pathway regulates p27 phosphorylation at threonin 187 as part of the mechanism triggered by early-weaning to induce cell proliferation in rat gastric mucosa. PloS One 8: e66651.

Tusher VG, Tibshirani R and Chu G (2001). Significance analysis of microarrays applied to the ionizing radiation response. Proc. Natl. Acad. Sci. U. S. A. 98: 5116-5121.

Wang M, Wang JJ, Li J, Park K, et al. (2009). Pigment epithelium-derived factor suppresses adipogenesis via inhibition of the MAPK/ERK pathway in 3T3-L1 preadipocytes. Am. J. Physiol. Endocrinol. Metab. 297: E1378-E1387. 